\title{
Erratum to: Assessing Cyanobacterial Harmful Algal Blooms as Risk Factors for Amyotrophic Lateral Sclerosis
}

\author{
Nathan Torbick ${ }^{1,2} \cdot$ Beth Ziniti $^{1} \cdot$ Elijah Stommel $^{2} \cdot$ Ernst Linder $^{3} \cdot$ Angeline Andrew $^{4}$. \\ Tracie Caller $^{4}$ - Jim Haney ${ }^{5}$. Walter Bradley ${ }^{6}$ - Patricia L. Henegan ${ }^{2} \cdot$ Xun Shi $^{7}$
}

\section{Erratum to: Neurotox Res (2017)}

$$
\text { https://doi.org/10.1007/s12640-017-9740-y }
$$

The article "Assessing Cyanobacterial Harmful Algal Blooms as Risk Factors for Amyotrophic Lateral Sclerosis", written by Nathan Torbick, was originally published electronically on the publisher's internet portal (currently SpringerLink) on May 13, 2017 without open access.

With the author(s)' decision to opt for Open Choice the copyright of the article changed on September 18, 2017 to
(C) The Author(s) 2017 and the article is forthwith distributed under the terms of the Creative Commons Attribution 4.0 International License (http://creativecommons.org/ licenses/by/4.0/), which permits use, duplication, adaptation, distribution and reproduction in any medium or format, as long as you give appropriate credit to the original author(s) and the source, provide a link to the Creative Commons license and indicate if changes were made.

The original article was corrected.

The online version of the original article can be found at https://dx.doi. org/10.1007/s12640-017-9740-y

Nathan Torbick

ntorbick@appliedgeosolutions.com

Applied Geosolutions, 55 Main St, Suite 125,

Newmarket, NH 03857, USA

2 Department of Neurology, Dartmouth College, Hanover, NH, USA

3 Department of Mathematics and Statistics, University of New Hampshire, Durham, NH, USA

4 Dartmouth-Hitchcock Medical Center, Lebanon, NH, USA

5 Department of Biological Sciences, University of New Hampshire, Durham, NH, USA

6 Department of Neurology, University of Miami Miller School of Medicine, Miami, FL, USA

7 Department of Geography, Dartmouth College, Hanover, NH, USA 